\title{
BLOOD AND ELECTROLYTE BALANCE IN THE POST-OPERATIVE CARDIAC PATIENT *
}

\author{
BY
}

\author{
M. V. BRAIMBRIDGE
}

(RECEIVED FOR PUBLICATION MAY 30, 1960)

A great deal has been written about postoperative fluid and electrolyte balance after abdominal operations. Relatively little attention has been paid to similar problems after cardiac surgery, particularly when extracorporeal circulation has been used. It may be valuable therefore to outline a post-operative régime used by most cardiac centres with minor modifications.

Consideration of blood and electrolyte balance after a cardiac operation may conveniently be divided into three sections: the maintenance of a normal blood volume, the problem of the unexpected blood pressure fall, and the necessity for supplying water and electrolyte requirements.

\section{Maintenance of Normal Blood Volume}

After cardiac surgery, the most important single factor in the immediate post-operative period is the maintenance of the normal blood volume. Too large a blood volume causes overloading of the ventricles, venous stasis, and pulmonary oedema. Too small a volume results in vasoconstriction, peripheral anoxia, and the picture of surgical shock.

In order to keep the volume constant, blood loss must be accurately replaced as it occurs. Blood is lost mainly into the thoracic cavity-pleura, pericardium, and mediastinum-though loss from thoracic and femoral wounds and blood withdrawn for haematological studies may assume significant proportions in children.

The immediate appearance of blood in calibrated chest drainage bottles is essential for its prompt replacement. These bottles should be tall and thin, so that small amounts of blood may be easily appreciated. A litre measuring cylinder is suitable if the top is cut off to take a bung, and the bottom anchored to a wider base, with $100 \mathrm{ml}$. of water for an underwater seal (Fig. 1).

${ }^{*}$ A lecture given at the Institute of Diseases of the Chest, Brompton Hospital, London.
Efficient drainage allows of immediate recognition of blood loss. Pleural drainage is commonplace in thoracic surgery and needs little mention, except to emphasize the importance of having the basal drain in the paravertebral gutter where blood accumulates. Tubes tend to swing forwards with the weight of their external length and it is often desirable to anchor the drain posteriorly inside the chest.

Pericardial drainage is more important than pleural. Tamponade is a common cause of death following cardiac surgery and accumulations too

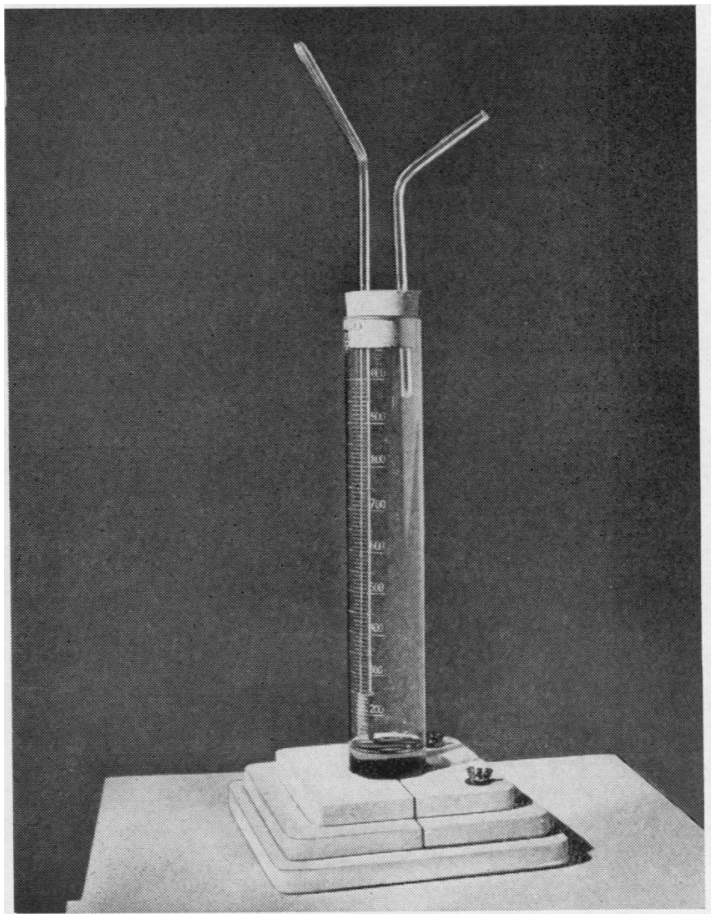

FIG. 1.-Thoracic cavity drainage bottle used at the Brompton Hospital, London. 
small to cause tamponade may result in troublesome tachycardia (Emanuel, 1960). Too much emphasis is often placed on the danger of dislocation of the heart if the pericardium is not carefully closed. Small drainage holes are then left that may be adequate for serious discharge but are quickly closed by haemorrhage of clotting blood. Dislocation of the heart rarely, if ever, occurs in the presence of intact lungs. The pericardium may therefore safely be left loosely closed or, better still, not closed at all. Even this is often inadequate after median sternotomy if the pleurae have not been opened, since mediastinal fat may seal off the pericardial defect. A pericardial drain is then a safety valve. Dowse catheters, size 8-11 F, are soft, do not irritate the heart, and drain efficiently. Anterior mediastinal drainage is also necessary after median sternotomy, since such collections of blood may mimic pericardial tamponade. Dowse catheters are equally useful for this purpose.

Drainage is assisted by suction and posture and checked by radiographs. Suction with a Roberts pump helps the passage of clots along the tubes. Posture, raising the shoulders on pillows and tipping the patient from side to side, allows gravity to bring pockets of blood into the lower paravertebral gutters, where the pleural drainage tubes lie. A radiograph of the chest is useful to exclude unexpected accumulations of blood clot. But radiographs may be both misleading and dangerous. If the film is taken with the patient lying flat a pint or more of blood may lie posteriorly without appearing on the film. A hypotensive patient may be killed if suddenly sat up by a radiographer. A good compromise is to take the film with the patient held at $45^{\circ}$ to the horizontal.

An accurate balance is maintained by efficient drainage and constant intravenous replacement.
The blood balance is checked mathematically and clinically. Blood loss at operation will have been estimated by weighing swabs and measuring the blood removed by suction, and will have been accurately replaced. At the end it may be again checked by weighing the patient. Post-operatively, a chart is kept of progressive totals of blood loss and replacement after the patient has returned to the ward. The use of such a chart is simple, easy to teach to nurses, and enables a balance to be constantly struck so that the rate of transfusion may be altered when necessary (Fig. 2). It is convenient to add a column to the post-operative balance chart, on which blood pressure, temperature, pulse and respiration rates are entered at the time that the mathematical balance is struck. The post-operative progress of the patient is then readily apparent from the chart alone.

Clinical assessment of the patient checks the mathematical balance. The arterial and venous pressures, pulse rate, and the state of the lung bases will suggest over- or under-transfusion.

The weight is another check on blood balance which is, at times, useful in the immediate postoperative period when there has been severe haemorrhage, or the patient has been returned to the operating theatre. Comparison with the preoperative weight enables an accurate balance to be resumed. Mathematics, however, should not be allowed to obscure the clinical picture. In the case of discrepancy between the two, clinical evidence should be preferred to mathematical. This must be so, for the mathematical balance depends on free drainage and if blood accumulates within the chest the observations are misleading.

Red blood cells, tagged with radioactive chromium, may also be used to estimate pre- and post-operative blood volumes. While being theoretically more accurate than the indirect

Date...............

POST-OPERATIVE FLUID CHART

Sheet No...............

Blood given in theatre:

Blood loss in theatre:

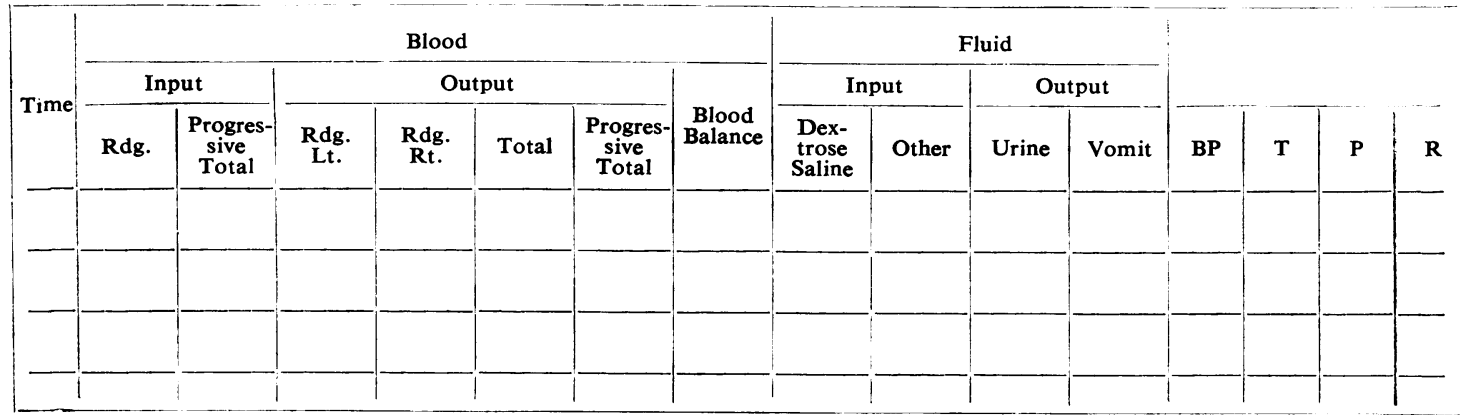

FIG. 2.-Post-operative fluid balance chart used at the Brompton Hospital, London. 
measurements, the technique is only possible in larger centres and is too complicated for routine use. It may be useful as an occasional check on the accuracy of the other methods.

\section{Unexpected Blood Pressure fall}

Attention to the régime described makes the post-operative course of the average cardiac patient straightforward and manageable by the nursing staff. Nevertheless the problem of an unexpected fall in blood pressure may occur. Haemorrhage is the immediate thought that arises, but there are at least six other conditions that may cause difficulty in diagnosis. These are cardiac failure, heart block, tamponade, vasodilatation, water and electrolyte imbalance, and adrenal exhaustion.

Unrecognized haemorrhage is the commonest cause of an unexpected blood pressure fall, and its frequency is proportional to the care that has been taken with haemostasis and drainage of the thoracic cavity. Haemostasis is particularly important if heparin has been used. Haemorrhage may occur with normal or with abnormal clotting. With normal clotting, haemorrhage may occur if a bleeding point has been left uncontrolled within the chest or if bleeding starts afresh. Haematological investigations will be normal, there will be clots in drainage tubes and bottles, and the chest wound will be dry. If bleeding is severe the patient must be returned to the operating theatre to secure proper haemostasis.

Failure to clot may be due to low levels of platelets or fibrinogen, the presence of fibrinolysins, or excessive use of anticoagulants such as heparin and "dindevan." Such bleeding may occur after any cardiac operation, normothermic, hypothermic, or extracorporeal. It is most frequent with deeply cyanosed patients after hypothermia where the circulation has been occluded longer than 10 minutes and after extracorporeal operations with inadequately cleaned or traumatic apparatus. It is recognized by an oozing thoracic wound, the complete absence of clots in chest drainage bottles and drains, and abnormal haematological studies. Most measurable clotting factors may be normal in the presence of an obvious bleeding state.

The differentiation of the type of haemorrhage is important. If haemostasis is at fault ligation of the bleeding vessel is essential. If there is abnormal clotting re-operation is useless and often dangerous. Re-opening the chest confronts the surgeon with widespread oozing and the patient is returned to bed with bleeding uncontrolled and sometimes with superadded cerebral symptoms.
Since detection of the clotting factor that is at fault is time consuming and often impossible, empirical treatment is necessary. If anticoagulants have been used before or during the operation, an extra dose of the appropriate antagonist is indicated. The triad of calcium, fibrinogen, and fresh blood will then be found to be effective, if used sufficiently early before the patient's blood volume has been replaced with stale, citrated, stored blood.

Enough calcium $(10 \mathrm{ml}$. of $10 \%$ calcium gluconate per pint of transfused citrated blood) is injected. A bottle of fibrinogen is reconstituted and given intravenously. A donor is obtained and a sample of blood taken so that cross-matching may be begun at once. The fresh blood is then taken into a plastic bag and the patient immediately transfused. It is useful to cross-match relatives in advance before a severe operation where bleeding is anticipated since they will be available quickly if required. The use of fibrinogen is unpopular with blood banks since it is costly and inconvenient to make. This disadvantage must be weighed against its sometimes dramatic action in stopping haemorrhage even when the fibrinogen level of the patient is apparently normal.

Cardiac failure is the main differential diagnosis of haemorrhage. It is vital to differentiate these two, as rapid transfusion is the treatment of the latter but may be fatal in the former. Tachycardia, a low blood pressure, and vasoconstriction are common to both, but pulmonary oedema and a raised jugular venous pressure will favour cardiac failure. In cases of difficulty, a therapeutic test with the rapid injection of a small amount of blood, some 50 to $100 \mathrm{ml}$, may be helpful. If the condition is failure there will be little response ; if due to haemorrhage the blood pressure usually rises sharply.

Heart block usually follows immediately after trauma to the bundle of His during closure of a septal defect. Complete heart block is recognized by a slow ventricular rate and cannon waves in the venous pressure. Continuous recording of the electrocardiogram on an oscilloscope has been found useful by both nurses and doctors post-operatively, since sudden alterations in rhythm or cardiac arrest are apparent and delay in treatment is minimal. Isoprenaline, 5-10 mg., may be given per rectum as often as is necessary to maintain an adequate heart rate. If this fails external electrodes may be placed over the heart and connected to a pacemaker. A higher voltage is necessary than when leads have been left in the heart, and twitching of the pectoral muscles, pain, and even burns distress 
the patient. The insertion of an insulated stainless steel wire into the ventricle through a wide-bore needle avoids these disadvantages.

Tamponade is a real hazard in cardiac surgery and minor degrees of it often go unrecognized, causing unexplained tachycardia and poor immediate post-operative progress. It is due to inadequate pericardial drainage. Measures to counteract this have already been outlined. Tamponade mimics cardiac failure, with tachycardia, low blood pressure, and a raised jugular venous pressure. A marked pulsus paradoxus has been found to be the best differentiating feature and has allowed immediate thoracotomy to avert disaster on more than one occasion.

Vasodilatation, particularly following extracorporeal circulation, sometimes causes a fall in blood pressure. A patient may return to the ward vasoconstricted, pale, with poor peripheral pulses. Some hours later he may suddenly vasodilate, become pink and warm, look much better but be markedly hypotensive. The reason for this vasodilatation is obscure, since it occurs even when the blood volume is normal, whether calculated by mathematical balance of blood loss, the patient's weight, or radioactive isotope studies. The treatment is blood transfusion in excess of theoretical requirements, with an amount that may vary from $300 \mathrm{ml}$. in children to $1,000 \mathrm{ml}$. in adults. With experience, this vasodilatory episode may be anticipated by maintaining a positive blood balance during the vasoconstrictive phase, so that, when the anticipated vasodilatation occurs, serious hypotension will be avoided.

Water and electrolyte imbalance does not cause a sudden drop in blood pressure, but may result in troublesome hypotension if oral intake is restricted for any reason, and care has not been taken to replace them. If the calculated inevitable loss of water is replaced as a routine post-operative measure, imbalance may be ignored in the diagnosis of a fall in blood pressure.

Adrenal exhaustion is a rare cause of postoperative hypotension. Sometimes, even when intravenous noradrenaline fails to raise the blood pressure, a good response may be obtained by an injection of cortisone. Consequently cortisone is often given during the treatment of obscure postoperative hypotension, but it is rare for it to be of proven value.

\section{Water and Electrolyte Requirements}

The necessity for post-operative blood balance is apparent. Less obvious is the importance of fluid balance. There are two policies that may be adopted in this respect. The estimated inevitable loss of water and electrolytes may be replaced as a routine, or they may be ignored and oral feeding relied upon to correct any discrepancy. The latter is the common practice in most thoracic surgical centres and works satisfactorily in the main. For cardiac cases, particularly after extracorporeal circulation operations, the maintenance of fluid balance is always important and sometimes vital. Oral feeding is not always resumed at once ; nausea, vomiting, or cerebral symptoms may cause further delay, and, if provision has not been made to cover each day's losses as they occur, a dangerous state of dehydration may result. Replacement of expected fiuid loss is simple, and will avert the hypotension and anuria of water and electrolyte depletion.

WATER.-There are few figures available for the amount of water inevitably lost by a patient following cardiac surgery. Careful studies have been made at the Mayo Clinic on the average losses of water following total heart/lung bypass operations (Sturtz, Kirklin, Burke, and Power, 1957). Water was lost as obligatory urine, insensible water loss from lungs and skin, and abnormal losses due to vomiting and thoracic drainage not replaced with blood. Against this was set the gain of water of oxidation and of catabolism of fat, carbohydrate, and protein, the so-called metabolic water (Table I).

TABLE I

POST-PERFUSION WATER LOSS (MAYO CLINIC)

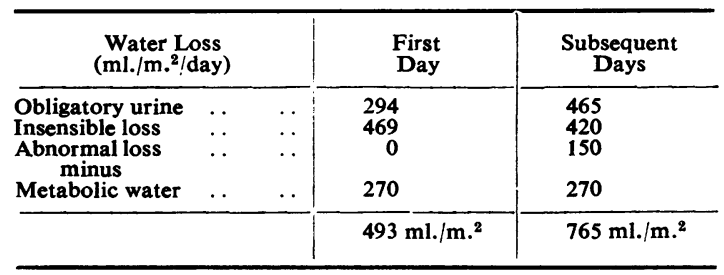

From these figures it can be seen that during the first 24 hours after operation some $500 \mathrm{ml}$. of water per square metre of surface area of the patient was lost. This is two-thirds of the loss on the second day that was some $750 \mathrm{ml}$. per sq. metre. The loss on the third day was almost identical.

Nurses change and fluid balances are drawn up at 8 a.m., so it is convenient to reckon a day as starting at this time. After a major cardiac operation, there are usually about 18 of the 24 hours remaining, that is, three-quarters of the day. Only two-thirds of the subsequent day's water requirements are needed on the first day, $\frac{2}{3} \times \frac{3}{4}=\frac{1}{2}$; so it may be said that $750 \mathrm{ml}$. of water per sq. 
metre will replace inevitable loss on the second and third days with half of this on the day of operation. At the Mayo Clinic, cold, humid oxygen tents were used post-operatively, and it is wise to increase this amount as a routine to $800 \mathrm{ml}$., with $400 \mathrm{ml}$. on the day of operation.

Sodium.-The question of whether cardiac patients should post-operatively be given sodium is difficult to answer dogmatically. On the one hand, it may be said that a patient in heart failure will retain sodium, which, combined with the normal post-operative sodium retention, will make further administration of it dangerous. On the other hand, most patients with any degree of heart failure will have been energetically treated with a low salt intake and diuretics. If too energetic a régime of dehydration has been adopted before the operation, sodium will definitely be required post-operatively.

Sodium is less obviously required when salt has not been restricted. At Stanford University Hospital, San Francisco, dextrose $5 \%$ was at one time given as intravenous fluid replacement following all cardiac surgery. Electrolyte imbalance, sometimes serious, tended to occur if there were any delay in the resumption of oral intake. Nausea, vomiting, and hypotension were the sequels and a vicious circle was begun.

Normal sodium requirements are $50 \mathrm{mEq}$. per sq. metre of surface area per day (Snively and Sweeney, 1956). Taking into consideration postoperative sodium retention, $35 \mathrm{mEq}$. per sq. metre was arbitrarily chosen as the amount to be given on the average post-operative day with half of this on the day of operation. On this régime, electrolyte imbalance ceased to be a problem.

Potassium.-Potassium is only rarely required following cardiac surgery. It is necessary when cerebral symptoms have prevented oral intake for more than 48 hours, or when too enthusiastic a course of dehydration has been carried out with chlorothiazide or hydroflumethiazide without potassium supplements.

ENERGY.-Energy requirements need to be covered, particularly in children, in order to prevent ketosis. Normal energy requirements are met by $50 \mathrm{~g}$. of carbohydrate per sq. metre of surface area per day, so $40 \mathrm{~g}$. is enough, with half of this amount on the day of operation.

Administration of Water and Electrolyte REQUIREMENTS.-On an average post-operative day $800 \mathrm{ml}$. of water, $35 \mathrm{mEq}$. of sodium, and $40 \mathrm{~g}$. of carbohydrate per sq. metre of surface area will cover the minimal needs of the cardiac patient without danger of overloading the circulation. Eight hundred millilitres of one-third normal saline in $5 \%$ dextrose contains these quantities of water, sodium, and energy. Half this amount, $400 \mathrm{ml}$. per sq. metre, may be given on the day of operation ending at $8 \mathrm{a} . \mathrm{m}$. the following morning. One-third normal saline is not a standard solution in Great Britain. It may be made up by dispensaries, or one-fifth normal saline in dextrose used. This will give $22 \mathrm{mEq}$. of sodium in the same 800 $\mathrm{ml}$. of solution, which is probably adequate. The surface area is read from the DuBois nomogram from the height and weight of the patient. The exact quantity of fluid necessary is calculated therefrom.

The route of administration is initially intravenous. A blood drip is always running in the post-operative cardiac patient, and a separate transfusion set for the dextrose/saline may be inserted into the side of the blood transfusion tubing. Whenever the blood balance is level, blood may be turned off and dextrose/saline continued instead. This is a positive advantage in post-operative nursing, since the blood does not have to be transfused so slowly that the cannula in the vein clots. The calculated quantity of fluid is given by 8 a.m. the following morning, both blood and fluid balance being maintained by relatively simple adjustments with the transfusions.

Oral feeding may be begun when convenient. Thirst is a common symptom after severe cardiac operations, and is particularly prominent after extracorporeal circulation when it is seldom relieved even by large quantities of water by mouth. These quantities given orally may cause vomiting with further disturbance of electrolyte balance, and it is wise to restrict oral intake on the day of operation to small amounts. If this adds up to a significant volume, the intravenous administration of fluid is curtailed by the same quantity.

The régime set out here, while based on that originally recommended by the Mayo Clinic for the post-operative care of their open heart cases, differs from it in two important respects. Oral fluid is not totally restricted during the immediate post-operative period, and sodium is given intravenously. Too literal following of the Mayo Clinic dicta resulted in severe electrolyte difficulties at Stanford University Hospital before it was realized that the restriction of oral fluid at the Mayo Clinic was due to the nausea of ether anaesthesia, and of sodium due to the relatively large amount transfused by the Gibbon pump oxygenator. 


\section{SUMMARY}

The normal blood volume is maintained in the post-operative cardiac patient by the accurate replacement of blood loss as it occurs. Efficient drainage of pleura, pericardium, and mediastinum ensures the prompt appearance of blood loss in calibrated chest drainage bottles. Accurate replacement is then simple.

Monitors of blood balance are mathematical, using a chart of progressive totals of blood loss and replacement; clinical, taking into account arterial and venous pressures and weight; and special, with radioactive isotope tagged red cells.

Unexpected hypotension occurs. While this is often due to unrecognized haemorrhage, associated with either normal or abnormal clotting factors, other conditions may cause difficulty in diagnosis. These are cardiac failure, heart block, tamponade, vasodilatation, water and electrolyte imbalance, and adrenal exhaustion.
Water and electrolyte balance may be little less important than blood balance. A simple régime, covering inevitable loss without circulatory overloading, is the administration of $800 \mathrm{ml}$. of onethird normal saline in $5 \%$ dextrose per sq. metre of surface area on an average post-operative day with half of this on the day of operation. Any delay in the start of oral feeding will not then result in dehydration.

I am particularly grateful to Dr. Frank Gerbode for stimulating my interest in this subject while working in his department as Heller Fellow in Surgery at Stanford University, San Francisco. I am also indebted to Sir Russell Brock for further opportunities to study this subject and for his help and encouragement in the preparation of this paper.

\section{REFERENCES}

Emanuel, R. (1960). Thorax, 15, 00.

Snively, W. D., Jr., and Sweeney, M. J. (1956). Fluid Balance Handbook for Practitioners. Charles C. Thomas, Springfield, Illinois.

Sturtz, G. S., Kirklin, J. W., Burke, E. C., and Power, M. H. (1957). Circulation, 16, 988. 